\title{
Aprendizaje Organizacional: Análisis de la Exploración y Explotación de Conocimiento en Medianas Empresas
}

\author{
Vanessa Pertuz ${ }^{(1)}$, Adith Pérez ${ }^{(1)}$, María L. Geizzelez ${ }^{(2)}$ y Adriana Vega(1) \\ (1) Universidad de Santander, Facultad de Ingenierías, Grupo de Investigación Nuevas Tecnologías-UDES. Valledupar, \\ Colombia.E-mail: van.pertuz@mail.udes.edu.co; adi.perez@mail.udes.edu.co; val13151008@mail.udes.edu.co \\ (2) Universidad Doctor Rafael Belloso Chacín. Miembro del comité académico del doctorado en Ciencias Mención \\ Gerencia. E-mail: mgeizzelez@urbe.edu.ve
}

Recibido Oct. 23, 2018; Aceptado Dic. 19, 2018; Versión final Feb. 7, 2019, Publicado Jun. 2019

\section{Resumen}

El objetivo del artículo es analizar las actividades para el aprendizaje organizacional en medianas empresas. El artículo analiza las actividades de exploración y explotación de conocimiento. La investigación considera un estudio de tipo descriptivo, de campo, no experimental. Se aplicó un cuestionario de recolección de datos a una muestra de 44 medianas empresas en Valledupar, Colombia. Los resultados destacan la presencia de actividades de exploración y explotación de conocimiento en las empresas analizadas. Adicionalmente, los resultados indican que no existen diferencias significativas en el desarrollo de estas actividades en las empresas objeto del presente estudio.

Palabras clave: explotación del conocimiento; exploración del conocimiento; aprendizaje organizacional; medianas empresas

\section{Activities for Organizational Learning in Medium-size Companies}

\begin{abstract}
The paper aims to analyze the activities for organizational learning in medium-size companies. This paper studies the activities of knowledge exploration and knowledge exploitation. The research considers a descriptive approach, of field type, and not experimental form. A data collection questionnaire was applied to 44 medium-sized companies in Valledupar, Colombia. The results highlight the strong presence of activities about knowledge exploration and knowledge exploitation in the companies analyzed. Additionally, the results indicate the absence of statistical differences in the development of these activities inside the companies analyzed in this work.
\end{abstract}

Keywords: knowledge exploration; knowledge exploitation; organizational learning; medium size companies 


\section{INTRODUCCIÓN}

De acuerdo con los planteamientos de Oviedo et al. (2014) las actividades básicas del aprendizaje organizacional son la exploración y la explotación del conocimiento. De esta manera, los autores Duarte y Castañeda (2013) conciben el aprendizaje organizacional como una tensión entre la explotación y la exploración. Asimismo, Acosta y Fischer (2013) definen la ambidestreza organizacional como el desarrollo simultáneo de las actividades de explotación y exploración. La literatura destaca la importancia de la ambidestreza, asociada a una estrategia para lograr la supervivencia y el crecimiento en las empresas, mediante el aprendizaje organizacional (Lee et al., 2018). Asimismo, diversos autores analizan la relación de la ambidestreza con la generación un éxito financiero sostenido (Gualandris et al., 2018), el rendimiento individual de los colaboradores (Hong et al., 2018) y la estructura de capital de capital de las organizaciones (Miglietta et al., 2018). Este ultimo, destaca que las empresas con una estrategia de ambidestreza logran un mayor apalancamiento en sus negocios (Miglietta et al., 2018). En consecuencia, las actividades de exploración y explotación resultan fundamentales para la adaptación y supervivencia de la empresa, la exploración se fundamenta en el desarrollo de estrategias mediante la búsqueda de nuevas oportunidades, mientras que las estrategias de explotación se asocian a la búsqueda de ventajas de carácter más inmediato (Nicolau et al., 2015). Adicionalmente, la adquisición de conocimientos está asociada positivamente con la explotación del conocimiento para obtener ventajas competitivas, mediante el desarrollo de nuevos productos, la diferenciación tecnológica y la eficiencia en los costos (Yli Renko et al., 2001). En el mismo sentido, los autores Bierly III et al. (2009) señalan que la capacidad de una empresa para adquirir y explotar el conocimiento externo es fundamental para lograr y mantener una ventaja competitiva. Más recientemente, los resultados de los estudios de Gonzalez y de Melo (2018) establecen que esta ventaja competitiva es el resultado del proceso innovador, que requiere la exploración y explotación del conocimiento.

De esta manera, De Oliveira et al. (2015), a partir de la revisión de literatura, indican que las capacidades de exploración y explotación son tópicos centrales del proceso de innovación de productos. Adicionalmente, los autores concluyen que el conocimiento que obtienen las organizaciones se divide en dos categorías: la exploración de nuevas posibilidades y la explotación de conocimientos previamente adquiridos. Así, Faccin y Balestrin (2018) señalan que la dinámica de investigación y desarrollo, orientada a la creación de conocimiento, se deriva de prácticas complementarias de exploración y explotación, emprendidas durante la vida útil de un proyecto interorganizativo, que desencadena un proceso ambidextro de innovación. Específicamente, el estudio de $\mathrm{Li}$ et al. (2018) analiza el efecto de la explotación y la exploración del conocimiento en la generación de innovaciones; los resultados demuestran que asignar un mayor esfuerzo a la exploración que a la explotación, es beneficioso para lograr innovaciones disruptivas, a pesar del riesgo de generar innovaciones particularmente pobres. Por su parte, poner el mismo énfasis en la exploración y explotación del conocimiento, no resulta particularmente efectivo para lograr innovaciones disruptivas; sin embargo, es la mejor estrategia para evitar innovaciones pobres. Igualmente, el estudio de Vargas et al. (2018) establece el efecto de los flujos de explotación y exploración en el resultado innovador.

De otro lado, Vasconcelos et al. (2018) establecen que la adquisición e integración del conocimiento en las organizaciones implican sucesivas iteraciones de codificación, abstracción y difusión del mismo, en relación con los conceptos de los productos, los requisitos del proceso y los enfoques de resolución de problemas; lo cual se relaciona con la capacidad de adoptar, simultáneamente, diferentes trayectorias de exploración y explotación. De esta manera, la exploración y la explotación están estrechamente vinculadas, debido a que la explotación, sin explorar nuevos conocimientos con el tiempo genera ineficacias dado el agotamiento tecnológico del mercado. En contraste, el enfoque exclusivo hacia las capacidades de exploración, conduce a un déficit en la relación entre los costos de experimentación y el volumen de negocios a partir de nuevos productos; por lo que se requiere equilibrar estas dos actividades (De Oliveira et al., 2015). Si bien, es fundamental el equilibrio entre los flujos de exploración y explotación para la sostenibilidad organizacional, las empresas deben elegir entre los dos, debido a sus limitados recursos (De Oliveira et al., 2015). Incluso, dada la competencia de recursos limitados, cada capacidad puede aplicarse a un área específica de la empresa o incorporar un equilibrio entre estas dos capacidades como clave para la supervivencia organizacional (Acosta y Fischer, 2013). En contraste, Slater et al. (2014) destacan la importancia de la ambidestreza organizacional, asociando la explotación con innovaciones incrementales, y a la exploración con el desarrollo de inteligencia de mercado para el desarrollo de innovaciones radicales consideradas la base del flujo de efectivo de la organización. Igualmente, Slater et al. (2014) conciben la capacidad de exploración y explotación como dos estilos de aprendizaje que impactan las innovaciones.

Así, Nicolau et al. (2015) destacan que la explotación y exploración ha dado lugar a una literatura prolija, incluyendo estudios sobre el aprendizaje organizacional, la renovación estratégica y la innovación tecnológica; no obstante, se ha llegado a un consenso para la medición de estas capacidades a partir de la escala propuesta por Atuahene (2005). Asimismo, los autores Nicolau et al. (2015) señalan que cada una de estas actividades requieren diferentes estructuras, procesos, estrategias, capacidades, culturas y aprendizajes, y consecuentemente, pueden tener diversos impactos en el desempeño de la organización. 
Contrastados los postulados de los autores, Duarte y Castañeda (2013), De Oliveira et al. (2015) y Oviedo et al. (2014), conciben la explotación y la explotación como actividades de aprendizaje organizacional. Además, otros autores como Acosta y Fischer (2013) y Slater et al. (2014) hacen referencia a la ambidestreza organizacional. Adicionalmente, De Oliveira et al. (2015) indican que el uso exclusivo de las capacidades de exploración genera un déficit en la relación entre los costos de experimentación y el volumen de negocios, mientras que el enfoque exclusivo de explotación genera agotamiento tecnológico. Los autores Slater et al. (2014) y Nicolau et al. (2015), coinciden en afirmar que la exploración se asocia con innovaciones radicales, mientras que la explotación con innovaciones incrementales. Por su parte, Slater et al. (2014) conciben la explotación y la exploración como dos estilos de aprendizaje que impactan la innovación y (Nicolau et al., 2015) indican existe consenso en la literatura para la medición de las capacidades de explotación y exploración mediante la escala propuesta por Atuahene (2005). De acuerdo con lo anterior, el presente artículo se fundamenta en la siguiente pregunta de investigación ¿Cuáles son las actividades de aprendizaje organizacional que desarrollan las medianas empresas de Valledupar Colombia? Para tal fin, se analiza la exploración y explotación del conocimiento como actividades de aprendizaje organizacional, considerando la categorización propuesta por Oviedo et al. (2014).

\section{ASPECTOS TEÓRICOS}

En la literatura se presentan diferentes aproximaciones en referencia a los modelos de explotación y exploración de conocimiento. A manera de ejemplo, los autores Miller et al. (2006) agregan los elementos de aprendizaje interpersonal y conocimiento tácito al modelo de explotación y exploración del conocimiento propuesto por March en la década de los noventa; según el cual, la sostenibilidad de la organización está asociada a su capacidad para mantener un balance entre la explotación y la exploración del conocimiento. Adicionalmente, los autores Bell y Cooper (2018) desarrollan un marco de referencia para la comprensión de la adquisición del conocimiento institucional y sus procesos de asimilación y explotación en el contexto de la internacionalización. La tabla 1 presenta la revisión de literatura realizada frente al concepto de exploración.

Tabla 1: Revisión de literatura del concepto de exploración.

\begin{tabular}{|c|c|}
\hline Autor & Concepto de exploración \\
\hline Nicolau et al. (2015) & $\begin{array}{l}\text { Se fundamenta en la experimentación con nuevas alternativas que tienen retornos } \\
\text { inciertos, y en algunos casos negativos. Se asocia con la búsqueda de conocimiento } \\
\text { distante y la innovación radical. Tiene como fin lograr la flexibilidad y la novedad en la } \\
\text { innovación de productos, mediante mayores niveles de variación y experimentación de } \\
\text { nuevas alternativas. }\end{array}$ \\
\hline Atuahene (2005) & $\begin{array}{l}\text { Refleja la habilidad de la organización para desarrollar nuevas habilidades, conocimientos } \\
\text { y procesos }\end{array}$ \\
\hline Acosta y Fischer (2013) & $\begin{array}{l}\text { Permite la obtención de innovaciones radicales, que se convierten en diseños } \\
\text { tecnológicos dominantes durante un cierto periodo. }\end{array}$ \\
\hline $\begin{array}{l}\text { Duarte y Castañeda } \\
\text { (2013) }\end{array}$ & $\begin{array}{l}\text { Es la asimilación de nuevos conocimientos, esta actividad se asocia con la búsqueda de } \\
\text { conocimientos nuevos para la organización. }\end{array}$ \\
\hline De Oliveira et al. (2015) & $\begin{array}{l}\text { La búsqueda de conocimientos y habilidades completamente nuevos en el desarrollo de } \\
\text { productos. }\end{array}$ \\
\hline Oviedo et al. (2014) & $\begin{array}{l}\text { Representa un flujo de conocimientos desde el individuo o grupo hacia la organización } \\
\text { para el desarrollo asimilación de conocimientos, que modifican las creencias y los } \\
\text { comportamientos del sistema organizacional. Los flujos de conocimiento de exploración } \\
\text { se direccionan hacia la renovación, la creación, la variación y el cambio. } \\
\text { Características: la búsqueda, la variación, el riesgo, la experimentación, la flexibilidad, el } \\
\text { descubrimiento y la innovación; su esencia es la experimentación de alternativas } \\
\text { novedosas, con resultados inciertos y distantes en el tiempo. }\end{array}$ \\
\hline Yalcinkay et al. (2007) & $\begin{array}{l}\text { Desde la perspectiva de las capacidades dinámicas, la define como la capacidad de la } \\
\text { empresa de adoptar nuevos procesos y productos únicos, diferentes de los utilizados en } \\
\text { el pasado. Se asocian con la sostenibilidad de la organización a largo plazo y debe } \\
\text { asociarse a la estrategia de la organización. }\end{array}$ \\
\hline Slater et al. (2014) & $\begin{array}{l}\text { La exploración se asocia con la búsqueda de información nueva y radical en el mercado, } \\
\text { que más allá del conocimiento del mercado actual. La exploración se asocia con } \\
\text { innovaciones radicales }\end{array}$ \\
\hline
\end{tabular}

Contrastando las definiciones presentadas en la literatura, Nicolau et al. (2015) asocian la exploración con la experimentación de alternativas con retornos inciertos. Paralelamente, Atuahene (2005), Duarte y Castañeda (2013), Oviedo et al. (2014), Yalcinkaya et al. (2007) y De Oliveira et al. (2015) la definen como el desarrollo de nuevas habilidades y la asimilación de nuevo conocimiento. Por su parte, autores como Acosta y Fischer (2013), Lloria y Moreno (2014) relacionan el concepto de exploración con el desarrollo de innovaciones 
radicales. En otro sentido, Lloria y Moreno (2014) establecen los siguientes reactivos para la medición de la capacidad de exploración: la capacidad de las personas para romper ideas tradicionales y ver las cosas desde una perspectiva diferente; la comprensión de los colaboradores de la forma como piensan y actúan sus compañeros de trabajo; la creación y unión de los grupos para crear soluciones radicalmente nuevas a los problemas; la comunicación entre colaboradores frente a temas pertinentes de sus áreas de trabajo; la consideración del punto de vista de todos los colaboradores en las reuniones; la existencia de sistemas de información para compartir conocimientos de los colaboradores y la existencia de mecanismos formales de buenas prácticas compartidas con diferentes departamentos. Asimismo, el dialogo en el grupo de trabajo; los procedimientos de para la gestión de ideas de los colaboradores; la comunicación de los nuevos desarrollos de la empresa, mediante reuniones e informes; el establecimiento de alianzas y redes con otras empresas para fomentar el aprendizaje y los acuerdos con universidades y centros tecnológicos. Adicionalmente, los resultados de Santoro y Usai (2018) confirman un efecto directo e importante de las prácticas de gestión de recursos humanos en la exploración del conocimiento. La tabla 2 presenta la revisión de literatura referente a explotación del conocimiento.

Tabla 2: Revisión de literatura del concepto de explotación.

\begin{tabular}{|c|c|}
\hline Autor & Concepto de explotación \\
\hline Nicolau et al. (2015) & $\begin{array}{l}\text { Se concentra en el perfeccionamiento, la ampliación de competencias existentes y las } \\
\text { tecnologías con retornos próximos y predecibles y generalmente positivos. Se asocia con } \\
\text { la búsqueda de conocimiento local y la innovación incremental. Su objetivo es perfeccionar } \\
\text { y ampliar conocimientos existentes mediante la inversión de recursos, la búsqueda local, } \\
\text { el refinamiento experimental, la selección y reutilización de las rutinas existentes para } \\
\text { mejorar la productividad y eficiencia de los recursos, reduciendo la variedad y reafirmando } \\
\text { las innovaciones existentes. }\end{array}$ \\
\hline Oviedo et al. (2014) & $\begin{array}{l}\text { Se asocia con la eficiencia, selección y ejecución. Se focaliza en la mejora y el uso del } \\
\text { conocimiento existente, adaptándose a las condiciones para generar un resultado } \\
\text { predecible y cercano en el tiempo. Genera un aprendizaje adaptativo con innovaciones } \\
\text { incrementales. Los flujos de explotación van desde la organización hacia los grupos o } \\
\text { individuos permitiendo la difusión, combinación y utilización de los conocimientos } \\
\text { existentes, enfatizando la convergencia, la retrospección, la institucionalización y la } \\
\text { estabilidad. }\end{array}$ \\
\hline Acosta y Fischer (2013) & $\begin{array}{l}\text { Permite la obtención de sucesivas innovaciones incrementales, mejorando algunos de los } \\
\text { atributos de los productos, hasta que se presente un cambio que genere un nuevo régimen } \\
\text { tecnológico }\end{array}$ \\
\hline $\begin{array}{l}\text { Duarte y Castañeda } \\
(2013)\end{array}$ & $\begin{array}{l}\text { uso del conocimiento previamente aprendido, generando la utilización continuo de la base } \\
\text { de conocimientos de la organización; esta actividad se basa en la búsqueda local, el } \\
\text { refinamiento de la experiencia y la reutilización de las rutinas existentes }\end{array}$ \\
\hline Yalcinkaya et al. (2007) & $\begin{array}{l}\text { Desde la perspectiva de las capacidades dinámicas, la define como la capacidad de la } \\
\text { empresa de mejorar continuamente sus recursos existentes y procesos. }\end{array}$ \\
\hline De Oliveira et al. (2015) & $\begin{array}{l}\text { Uso y perfeccionamiento de conocimientos y habilidades en el desarrollo de productos } \\
\text { existentes. Se asocia a la utilización efectiva de los dominios tecnológicos y de mercado } \\
\text { existentes en la empresa, mejorando la eficiencia, reduciendo la incertidumbre en el } \\
\text { proceso y generando retornos positivos, próximos y predecibles }\end{array}$ \\
\hline Slater et al. (2014) & $\begin{array}{l}\text { La explotación se centra en el aprendizaje por perfeccionamiento y ampliación de la } \\
\text { información existente, esta se asocia con innovaciones incrementales y con el } \\
\text { mejoramiento de la rentabilidad. }\end{array}$ \\
\hline
\end{tabular}

De otro lado, Lloria y Moreno (2014) establecen los siguientes reactivos para medición de la explotación de conocimiento: la presencia de los procedimientos, manuales, normas y procesos establecidos en la organización y la existencia de bases de datos para almacenar las experiencias y conocimientos de los colaboradores. Por su parte, Nicolau et al. (2015) indican que, para el análisis de la capacidad de explotación, es fundamental conocer si la organización mantiene y mejora sus conocimientos y habilidades en tecnologías y procesos misionales de la misma; dado que esta representa la habilidad para refinar y extender sus habilidades, conocimientos y procesos existentes previamente, lo que permite mejorar la eficiencia de las innovaciones organizacionales. En suma, se evidencia consenso frente al hecho de que el concepto de explotación, se asocia con el uso y perfeccionamiento del conocimiento existente con retornos predecibles y generalmente positivos, considerando los postulados de autores como Nicolau et al. (2015), Oviedo et al. (2014), Yalcinkaya et al. (2007), De Oliveira et al. (2015) y Duarte y Castañeda (2013). Por su parte, Lloria y Moreno (2014) relacionan la explotación con los procedimientos institucionales y la existencia de bases de datos de conocimiento de los colaboradores. Asimismo, Acosta y Fischer (2013) vincula la explotación con el desarrollo de innovaciones incrementales. 


\section{METODOLOGÍA}

La investigación se enmarca en la escuela de pensamiento del positivismo lógico (Briones, 1996), con un enfoque cuantitativo. La investigación se aborda desde un diseño de campo (Tamayo, 2011); de tipo no experimental y transaccional (Hernandez et al., 2014). La investigación utiliza la encuesta como técnica de recolección de datos (Martínez Ruiz, 2012), mediante un cuestionario de preguntas cerradas (Hernandez et al., 2014), el cual fue diseñado en el marco de la presente investigación. A continuación, se presenta la ficha correspondiente al proceso de recolección de datos (Tabla 3):

Tabla 3: Ficha técnica del proceso de recolección de datos.

\begin{tabular}{|c|l|}
\hline Característica & Descripción \\
\hline Características de los informantes & Gerentes, jefes de talento humano, coordinadores de área. \\
\hline Fecha de aplicación & Agosto - diciembre de 2017 \\
\hline Metodología de aplicación & Visitas a los informantes claves en las empresas. \\
\hline $\mathrm{N}^{\circ}$ de ítems del instrumento & Escala validada compuesta por 6 ítems \\
\hline Población & $\begin{array}{c}\text { Medianas empresas del municipio de Valledupar, Cesar, Colombia, legalmente } \\
\text { constituidas y con domicilio comercial en Valledupar, correspondiente a un total } \\
\text { de 81 empresas, de acuerdo con la Cámara de Comercio de Valledupar. }\end{array}$ \\
\hline \multirow{2}{*}{ Estimación de la muestra } & $\begin{array}{l}\text { Muestreo probabilístico, de tipo aleatorio simple. } \\
\text { Z }=\text { Nivel de confianza (Z = 1.96 para una confianza del 95\%) } \\
\text { E = Margen de error seleccionado (E = 10\%) } \\
\text { De acuerdo con la fórmula para el cálculo del tamaño óptimo de la muestra en } \\
\text { poblaciones finitas propuesta por Martínez (2012), se obtiene una muestra de 44 } \\
\text { medianas empresas. }\end{array}$ \\
\hline
\end{tabular}

El cuestionario incluye una escala tipo Likert con 5 opciones de respuesta: 5: Siempre, 4: Casi siempre, 3: Algunas veces, 2: Casi nunca, 1: Nunca. La validez del cuestionario se determinó mediante juicio de expertos. La confiabilidad se estimó mediante el coeficiente de alfa de Cronbach (Hernandez et al., 2014), mediante la aplicación del instrumento a empresas, mediante una prueba piloto, obteniendo un valor de 0,921 , lo que indica que el instrumento es confiable. Es relevante destacar que, la elección de la técnica estadística está determinada, entre otros factores, por el nivel de medición de las variables. En el presente artículo, se utiliza la escala de medición tipo Likert. Al respecto, Hernández et al., (2014, p. 243) establecen que "la escala de Likert es, en sentido estricto, una medición ordinal sin embargo, es común que se le trabaje como si fuera de intervalo". Consecuentemente, el presente trabajo utiliza la estadística descriptiva, específicamente, la media y la desviación estándar para determinar la presencia de actividades de exploración y explotación. Para la interpretación de los resultados de la estadística descriptiva se estableció un punto de comparación o baremo de interpretación; el cual fue construido, para efectuar el proceso de análisis de los resultados. El baremo de la media (Tabla 4) se establece desde 1 hasta 5 debido a que corresponden al valor mínimo y máximo de la escala de respuesta del cuestionario.

Tabla 4: Baremo para el análisis de las medias.

\begin{tabular}{|c|l|c|}
\hline Intervalos & Categorías & Convención \\
\hline $4,20-5,00$ & Muy presente & MP \\
\hline $3,40-4,19$ & Presente & $\mathrm{P}$ \\
\hline $2,60-3,39$ & Medianamente presente & MEP \\
\hline $1,80-2,59$ & Poco presente & $\mathrm{PP}$ \\
\hline $1,00-1,79$ & Ausente & $\mathrm{A}$ \\
\hline
\end{tabular}

Adicionalmente, se establece el baremo de interpretación con el valor mínimo y máximo de la desviación estándar (Tabla 5), obtenidos de la tabulación de los ítems del instrumento de recolección de datos.

Tabla 5: Baremo para el análisis de la desviación estándar.

\begin{tabular}{|c|l|c|}
\hline Intervalos & Categorías & Convención \\
\hline $1,39-1,74$ & Muy alta dispersión & MAD \\
\hline $1,05-1,38$ & Alta dispersión & ALD \\
\hline $0,70-1,04$ & Dispersión intermedia & DI \\
\hline $0,35-0,69$ & Baja dispersión & BD \\
\hline $0,00-0,34$ & Ausente dispersión & AD \\
\hline
\end{tabular}




\section{RESULTADOS Y DISCUSIÓN}

La tabla 6 presenta los resultados correspondientes a la exploración y explotación del conocimiento. Los resultados de la actividad de exploración indican que el $48 \%$ de las respuestas de los informantes se ubican en la opción siempre, el $25 \%$ de las respuestas en la opción casi siempre, para la alternativa algunas veces se obtiene el $18 \%$, mientras que para la alternativa nunca se registra un $7 \%$, y en la alternativa casi nunca un $2 \%$ de las respuestas de los informantes. Estos resultados indican que el indicador exploración se encuentra presente de acuerdo con las respuestas de los informantes claves, con una media de 4,03 de acuerdo con el baremo establecido para el análisis de datos (Tabla 4). Asimismo, se obtiene una desviación estándar de 1,18, lo que indica una alta dispersión de las respuestas de acuerdo con el baremo de análisis de la desviación estándar (Tabla 5).

Tabla 6: Resultados de las actividades para el aprendizaje organizacional.

\begin{tabular}{|c|c|c|c|c|c|c|c|c|c|c|c|c|c|c|c|c|}
\hline \multirow{3}{*}{ Actividad } & \multicolumn{10}{|c|}{ Opciones de respuesta } & & & \multirow{3}{*}{\multicolumn{2}{|c|}{$\bar{X}$}} & & \\
\hline & \multicolumn{2}{|c|}{ Nunca } & \multicolumn{2}{|c|}{$\begin{array}{c}\text { Casi } \\
\text { nunca }\end{array}$} & \multicolumn{2}{|c|}{$\begin{array}{l}\text { Algunas } \\
\text { veces }\end{array}$} & \multicolumn{2}{|c|}{$\begin{array}{c}\text { Casi } \\
\text { siempre }\end{array}$} & \multicolumn{2}{|c|}{ Siempre } & \multicolumn{2}{|c|}{ Total } & & & \multirow{2}{*}{\multicolumn{2}{|c|}{$\sigma$}} \\
\hline & $\mathrm{Fa}$ & $\%$ & $\mathrm{Fa}$ & $\%$ & $\mathrm{Fa}$ & $\%$ & $\mathrm{Fa}$ & $\%$ & $\mathrm{Fa}$ & $\%$ & $\mathrm{Fa}$ & $\%$ & & & & \\
\hline Exploración & 3 & 7 & 1 & 2 & 8 & 18 & 11 & 25 & 21 & 48 & 44 & 100 & 4,03 & $P$ & 1,18 & ALD \\
\hline Explotación & 3 & 7 & 4 & 9 & 9 & 20 & 12 & 27 & 16 & 36 & 44 & 100 & 3,82 & $P$ & 1,21 & ALD \\
\hline \multicolumn{13}{|c|}{ Media $(\bar{X})$ general } & \multicolumn{4}{|c|}{3,92} \\
\hline \multicolumn{13}{|c|}{ Categoría general de la media $(\bar{X})$} & \multicolumn{4}{|c|}{ Presente $(\mathrm{P})$} \\
\hline \multicolumn{13}{|c|}{ Desviación estándar $(\sigma)$ general } & \multicolumn{4}{|c|}{1,20} \\
\hline \multicolumn{13}{|c|}{ Categoría general de la desviación estándar $(\sigma)$} & \multicolumn{4}{|c|}{ Alta dispersión (ALD) } \\
\hline
\end{tabular}

Como elementos de discusión para los resultados obtenidos en las actividades de exploración, Acosta y Fischer (2013) destacan que la exploración implica la realización de actividades orientadas a la innovación, la búsqueda de novedades con los riesgos derivados y el descubrimiento de nuevas oportunidades. En el mismo sentido, Oviedo et al. (2014) señalan que la exploración representa la asimilación de nuevos conocimientos en la empresa direccionados al cambio. En este orden de ideas, De Oliveira et al. (2015) concluyen que las actividades de exploración mejoran la capacidad de innovación del producto debido al proceso acumulativo de nuevos conocimientos que se añade a la base de conocimientos de los colaboradores de la empresa; generando diferenciación de los productos y competitividad; sin embargo, las actividades de exploración en ocasiones presentan retornos inciertos, distantes en el tiempo, e incluso, negativos.

En segunda instancia, los resultados de la actividad de explotación evidencian que el 36\% de las respuestas de los informantes claves se ubica en la opción siempre, el 27\% de las respuestas en la opción casi siempre; para la alternativa algunas veces se obtiene el $20 \%$, mientras que para la alternativa casi nunca registra un $9 \%$, y para la opción nunca se obtiene un $7 \%$ de las respuestas de los informantes claves. En general, la actividad de explotación registra una media de 3,82, indicando que esta actividad se encuentra presente en las empresas analizadas, de acuerdo con el baremo establecido (Tabla 4). Asimismo, para la actividad de explotación del conocimiento se obtiene una desviación estándar de 121, lo que indica una alta dispersión de las respuestas, de acuerdo con el baremo de interpretación (Tabla 5).

En referencia a la discusión de la teoría correspondiente a las actividades de explotación, los autores De Oliveira et al. (2015) validan la hipótesis de que la explotación influencia positivamente la innovación de productos y el desempeño general. Adicionalmente, Soetanto y Jack (2018) analizan las capacidades de exploración y explotación en el contexto de las pequeñas empresas de base tecnológica, destacando que, pese a los recursos limitados, este tipo de empresas pueden realizar innovaciones basadas en los flujos de explotación y exploración de conocimiento. De la misma manera, Oviedo et al. (2014) destacan que la explotación se asocia con la eficiencia, selección y ejecución basado en la utilización y mejora del conocimiento existente; estas actividades generan resultados predecibles y cercanos en el tiempo, representados en innovaciones incrementales. De este modo, la explotación implica el perfeccionamiento de tecnología disponible, el aprender mediante la acción, la mejora en la división del trabajo y las actividades orientadas a la eficiencia (Acosta y Fischer, 2013). Considerando estos resultados, la media general de las actividades de aprendizaje organizacional corresponde a 3,92, ubicándose en la categoría presente, de acuerdo al baremo establecido (Tabla 4). Adicionalmente, la desviación estándar de las actividades de aprendizaje organizacional se ubica en 1,20 lo que indica una alta dispersión en las respuestas de acuerdo con el baremo (Tabla 5).

En suma, los resultados del presente artículo validan la presencia de las actividades de explotación y exploración del conocimiento, lo que denota ambidestreza organizacional. Al respecto, Acosta y Fischer 
(2013) señalan que el mantenimiento de un equilibrio entre la exploración y la explotación es una condición básica para la supervivencia y el éxito competitivo de una empresa, que implica el intercambio entre el conocimiento del entorno y el existente en la organización para generar cambios. Así, la ambidestreza organizacional facilita la capacidad de innovación y la rentabilidad de los conocimientos, evitando la obsolescencia (Acosta y Fischer, 2013). No obstante, Acosta y Fischer (2013) agregan que, aunque la exploración y explotación son procesos esenciales, estas actividades compiten por recursos escasos, por lo que algunas prácticas pueden resultar incompatibles entre sí; por lo que se sugiere encontrar una combinación optima en las diferentes áreas de la empresa. De este modo, los flujos de conocimiento tanto de explotación como de exploración, son los componentes de la capacidad de aprendizaje organizacional con mayor impacto en el desempeño empresarial (Oviedo et al., 2014). Al respecto, en el contexto de las pequeñas y medianas empresas el trabajo de Kilpi et al. (2018) señala la importancia del recurso de conocimiento como un factor explicativo del rendimiento de las organizaciones, asociando la exploración con el conocimiento del mercado de las organizaciones.

Específicamente, los resultados del presente artículo evidencian que la exploración se encuentra presente como actividad de aprendizaje organizacional en las empresas analizadas, lo cual indica que estas experimentan con alternativas novedosas, promoviendo el descubrimiento de conocimientos e implementando productos completamente nuevos en el mercado. Este hecho indica que las medianas empresas se orientan al aprendizaje mediante la búsqueda y el desarrollo de nuevos conocimientos. Igualmente, la explotación se encuentra presente en las medianas empresas, debido a que estas se focalizan en el uso del conocimiento existente, el desarrollo de proyectos con resultados predecibles y cercanos en el tiempo. Así, las medianas empresas combinan la exploración y la explotación, no obstante, estas actividades se encuentran presentes, resultado que corresponde a la segunda categoría del baremo establecido (Tabla 4) por lo que es fundamental implementar actividades orientadas al fortalecimiento de las mismas. De acuerdo con lo anterior, a continuación se proponen actividades que permiten fortalecer las competencias de ambidestreza organizacional en medianas empresas: en primer lugar, socializar los documentos y la base de conocimientos disponible en la organización, en segundo lugar, desarrollar proyectos con equipos de trabajo conformados por equipos de trabajo interno y personal externo para el desarrollo de procesos en la organización o la gestión de proyectos, en tercer lugar, apoyar los procesos de investigación y desarrollo (I+D) para proyectos de experimentación, en cuarto lugar, desarrollar convenios con universidades y centros de investigación y desarrollo, finalmente, revisar bases de datos científicas y patentes para la obtención de conocimientos desarrollados en procesos de investigación.

\section{CONCLUSIONES}

A partir de los resultados del presente artículo, se concluye la presencia de actividades de exploración y explotación del conocimiento. Específicamente, las actividades de exploración evidenciadas en las medianas empresas corresponden a la experimentación frente a alternativas novedosas, el descubrimiento de nuevos conocimientos y la implementación de productos completamente nuevos en el mercado. Por su parte, las actividades de explotación desarrolladas por las empresas objeto de estudio se asocian con el uso del conocimiento al interior de la organización y el desarrollo de resultados predecibles y cercanos en el tiempo. De esta manera, se evidencia la presencia de ambidestreza organizacional, debido a la combinación de actividades de explotación y exploración.

\section{REFERENCIAS}

Acosta Prado, J. C. y A. L. Fischer, Condiciones de la gestión del conocimiento, capacidad de innovación y resultados empresariales, Un modelo explicativo, Pensamiento y gestión, (35), 25 - 63 (2013)

Atuahene Gima, K., Resolving the capability-rigidity paradox in new product innovation, Journal of Marketing, 69(4), 61$83(2005)$

Bell, V.A. y S.Y. Cooper, Institutional knowledge: Acquisition, assimilation and exploitation in internationalization, International Marketing Review, 35(3), 475-497 (2018)

Bierly III, P.E., F. Damanpour y M. D. Santoro, The application of external knowledge: Organizational conditions for exploration and exploitation, Journal of Management Studies, 46(3), 481-509 (2009)

Briones, G., Epistemología de las ciencias sociales, Instituto Colombiano para el Fomento de la Educación Superior, ICFES, ARFO Editores e Impresores Ltda, Bogotá, Colombia (1996)

De Oliveira Cabral, J. E., A. F. Matos Coelho, F. J. Fernandes Coelho y M. D. Braga Costa, Capabilities, innovation, and overall performance in brazilian export firms, RAM. Revista de Administração Mackenzie, 16(3), 76-108 (2015)

Duarte-Aponte, S. P. y D. I. Castañeda Zapata, A model of organizational learning in practice, Estudios Gerenciales, 29(129), 439-444 (2013) 
Faccin, K. y A. Balestrin, The dynamics of collaborative practices for knowledge creation in joint R\&D projects, Journal of Engineering and Technology Management - JET-M, 48, 28-43 (2018)

Gonzalez, R. V. y T. M. de Melo, The effects of organization context on knowledge exploration and exploitation, Journal of Business Research, 90, 215-225 (2018)

Gualandris, J., H. Legenvre y M. Kalchschmidt, Exploration and exploitation within supply networks: Examining purchasing ambidexterity and its multiple performance implications, International Journal of Operations and Production Management, 38(3), 667-689 (2018)

Hernández, S.R., C. Fernandez y P. Baptista, Metodología de la investigación, 4ª Ed., McGrawHill, México, D.F., (2014)

Hong, K., G. Yu y E. Hyun, Understanding ambidexterity at the individual level: task assignment perspective, Computational and Mathematical Organization Theory, 24(1), 34-50 (2018)

Kilpi, V., H. Lorentz, T. Solakivi y J. Malmsten, The effect of external supply knowledge acquisition, development activities and organizational status on the supply performance of SMEs, Journal of Purchasing and Supply Management, 24(3), 247-259 (2018)

Lee, S.U., P. Gunno y K. Jina, The double-edged effects of the corporate venture capital unit's structural autonomy on corporate investors' explorative and exploitative innovation, Journal of Business Research, 88, 141-149 (2018)

Li, D., J. Lin, W. Cui y Y. Qian, The trade-off between knowledge exploration and exploitation in technological innovation, Journal of Knowledge Management, 22(4), 781-801 (2018)

Lloria, M. B. y M. D. Moreno Luzon, Organizational learning: Proposal of an integrative scale and research instrument, Journal of Business Research, 67(5), 692-697 (2014)

Martínez-Bencardino, C., Estadística y muestro, 13aㅗ, Ed., Ecoe Ediciones, Bogotá, Colombia (2012)

Martínez-Ruiz, H., Metodología de la investigación. Con el enfoque en competencias, $1^{a}$ Ed., Cengage Learning Editores, S.A. de C.V., México, D.F. México (2012)

Miglietta, N., E. Battisti, E. Carayannis y A. Salvi, Capital structure and business process management: evidence from ambidextrous organizations, Business Process Management Journal, 24(5), 1255-1270 (2018)

Miller, K. D., M. Zhao y R. J. Calantone, Adding interpersonal learning and tacit knowledge to March's explorationexploitation model, Academy of Management Journal, 49(4), 709-722 (2006)

Nicolau-Juliá, D., M. Expósito Langa y J.V. Tomás Miquel, Exploración y explotación de conocimiento en el ámbito empresarial. Validación de escalas en un sector industrial de bajo perfil tecnológico, Investigaciones Europeas de Dirección y Economía de la Empresa, 21(3), 139-147 (2015)

Oviedo-García, M. Á., M. Castellanos-Verdugo, A. Riquelme-Miranda y J. García del Junco, La relación entre aprendizaje organizacional y los resultados en la Administración Pública, Revista Europea de Dirección y Economía de la Empresa 23(1), 1-10 (2014)

Santoro, G. y A. Usai, Knowledge exploration and ICT knowledge exploitation through human resource management: A study of Italian firms, Management Research Review, 41(6), 701-715 (2018)

Slater, S. F., J. J. Mohr y S. Sengupta, Radical Product Innovation Capability: Literature Review, Synthesis, and Illustrative Research Propositions, Journal of Product Innovation Management, 31(3), 552-566 (2014)

Soetanto, D. y S. Jack, Slack resources, exploratory and exploitative innovation and the performance of small technologybased firms at incubators, Journal of Technology Transfer, 43(5), 1213-1231 (2018)

Tamayo, M., El proceso de la investigación científica, 5ํㅡㄹ. Ed., Limusa S.A., México D.F., México (2011)

Vargas, N., M. Begoña Lloria, A. Salazar y L. Vergara, Effect of exploitation and exploration on the innovative as outcomes in entrepreneurial firms, International Entrepreneurship and Management Journal, 1-17 (2018)

Vasconcelos, A., J. Martins, D. Ellis y E. Fontainha, Absorptive capacity: A process and structure approach, Journal of Information Science, (2018)

Yalcinkaya, G., T. J. Calantone y D. A. Griffith, An examination of exploration and exploitation capabilities: implications for product innovation and market performance, Journal of International Marketing, 15(4), 63-93 (2007)

Yli-Renko, H., E. Autio y H. J. Sapienza, Social capital, knowledge acquisition, and knowledge exploitation in young technology-based firms, Strategic Management Journal, 22(6-7), 587-613 (2001) 